\title{
LA REFLEXIÓN EN EL APRENDIZAJE-SERVICIO UNIVERSITARIO EN ACTIVIDAD FÍSICA Y DEPORTE. CLAVES PARA EL APRENDIZAJE PERSONAL, ACADÉMICO Y PROFESIONAL ${ }^{1}$
}

\author{
María Luisa Santos-Pastor \\ L. Fernando Martínez-Muñoz \\ Ignacio Garoz-Puerta \\ Luis García-Rico \\ Universidad Autónoma de Madrid
}

\begin{abstract}
RESUMEN: Este artículo analiza los procesos reflexivos que tiene el alumnado en el diseño, desarrollo y evaluación de proyectos de AprendizajeServicio Universitario. Estos procesos incrementan el aprendizaje y las competencias del alumnado, se logran mejores niveles de éxito, así como un cambio de mirada hacia la comunidad y los colectivos receptores que presentan situaciones de desventaja social. El estudio que se presenta utiliza como técnica de investigación el análisis de contenido. La información se recoge mediante cuestionarios reflexivos empleados en la evaluación formativa de los proyectos Aprendizaje-Servicio en Actividad Física y el Deporte de la Universidad Autónoma de Madrid. Los resultados muestran evidencias claras sobre la utilidad de los procesos reflexivos para hacer consciente al estudiante de lo que aprende y lo que necesita para aprender, a partir de la revisión crítica y consciente de lo que hace y aporta a la comunidad. El ámbito de la formación, la adquisición de competencias específicas y transversales son las dimensiones que más protagonismo tienen para el alumnado, mientras que las vinculadas con el escenario profesional son escasas. El estudio presenta limitaciones porque las reflexiones se extraen de un solo instrumento, pudiendo complementar los hallazgos con otras técnicas (diarios, informes de aprendizajes, etc.).
\end{abstract}

PALABRAS CLAVE: aprendizaje-servicio, actividad física, educación superior, reflexión.

1. Con el apoyo del Consejo Superior de Deportes de España (Ministerio de Cultura y Deporte), a través del Proyecto RIADIS (Red de Investigación en Servicio-Aprendizaje en Actividad Física y Deporte para la Inclusión Social-19 / UPB / 19) y 03/UPB/20. 


\title{
REFLECTION IN UNIVERSITY SERVICE-LEARNING IN PHYSICAL ACTIVITY AND SPORT. KEYS TO PERSONAL, ACADEMIC AND PROFESSIONAL LEARNING
}

\begin{abstract}
This article analyses the self-reflection processes of the students who participate in the design, development and assesment of the University ServiceLearning projects. These self-reflection processes increase student's learning and competencies; it is achieved better levels of success, as well as a change of view towards the community and the receiving groups which generally present situations of social disadvantage. The study presented is conducted by using content analysis as a research technique. The information is collected through the reflective questionnaires used in the educational evaluation of the Service-Learning projects in Physical Activity and Sport of the Autonomous University of Madrid. The results show clear evidence on the usefulness of reflective processes to make the students aware of what they learn and what they need to learn from a critical and conscious review of what they do and contribute to the community. The field of training and the acquisition of specific and cross-curricular competencies are the type of dimensions that hold the most prominence for students; otherwise those related to the professional setting are scarce. The study has limitations because the reflections are extracted from a single instrument, being able to complement the findings with other techniques (diaries, learning reports, etc.).
\end{abstract}

KEYWORDS: Service-learning, physical activity and sport, higher education, reflection.

Recibido: 30/03/2020

Aceptado: 17/04/2020

Correspondencia: María Luisa Santos-Pastor, Facultad de Formación de Profesorado y Educación. Universidad Autónoma de Madrid. Ciudad Universitaria de Cantoblanco. 28049 Madrid. Email: marisa.santos@uam.es

\section{INTRODUCCIÓN}

El Aprendizaje-Servicio Universitario (ApSU) se ha integrado en el tejido educativo como una oportunidad para resolver ciertas carencias de la formación inicial. Los aprendizajes del alumnado, en muchas ocasiones, no se han planificado para que sean transferibles a su profesión, ni tan siquiera se ha contemplado cómo prepararle para poder responder a las necesidades y exigencias de una sociedad en permanente cambio (Fandos, Renta, Jiménez y Gonzáles, 2017; Tejada-Fernández y Ruiz-Bueno, 2016; Wang, Lin, Spalding, Klecka y Odell, 2011).

El Espacio Europeo de Educación Superior (EEES) trajo consigo, entre otros cambios, la reorganización de los contenidos o saberes a trasmitir, proponiendo una enseñanza (que no siempre aprendizaje) más contextualizada en la vida real y capaz de responder (y 
solventar) a los grandes problemas existentes en el mundo (sostenibilidad, desigualdades, etc.). En este nuevo marco sociocultural y político, las universidades deben hacer frente a los desafíos europeos, reforzando la excelencia en materia de investigación y de formación (enseñanza). En este marco, los Objetivos de Desarrollo Sostenibles (ODS) propuestos por la Agenda 2030 constituyen una excelente guía para orientar y responder con acierto a la necesidad de lograr una mayor justicia social desde el ámbito universitario.

De este modo, todo parece indicar que la formación universitaria debe replantearse para favorecer un aprendizaje auténtico, empleando enfoques formativos abiertos a la comunidad y a la sociedad, proponiendo metodologías alternativas (activas) a las que tradicionalmente se han venido utilizando. Se demandan modelos formativos conectados con la vida, centrados en el desarrollo de competencias, en la construcción de un aprendizaje autónomo y en la adquisición de sus capacidades personales (competencia de aprender a aprender), promoviendo ciudadanos críticos y comprometidos con la sociedad (competencia social y cívica). La formación inicial precisa un nuevo enfoque didáctico, donde el alumnado tenga mayor protagonismo (Romero, Zurita-Ortega y Zurita-Molina, 2010) y pueda adquirir habilidades cognitivas, sociales, emocionales y éticas, transferibles a su contexto personal, social y profesional (Pagalagar, 2018, p. 330). Se trata de enfocar la formación hacia el "saber ser" de su profesión, proponiendo un aprendizaje basado en competencias que ofrezca contextos formativos o situaciones reales donde poner en práctica esos aprendizajes, y lo haga de manera efectiva, logrando un alto nivel de desempeño (Capraro, Capraro y Helfeldt, 2010).

En este contexto, el ApSU da respuesta a estos propósitos formativos, porque promueve un aprendizaje experiencial y una formación conectada con la realidad social. Desde este enfoque metodológico, el aprendizaje del alumnado se genera en ciclos de acción y de reflexión, trabajando en grupo y aplicando en la comunidad lo que han aprendido (Capella-Peris, Gil-Gómez y Chiva-Bartoll, 2019; Chiva-Bartoll, Pallarés-Piquer y Gil-Gómez, 2018; Chiva-Bartoll, Ruiz-Montero, Martín-Moya, Pérez-López, Giles-Girela, García-Suárez y Rivera-García, 2019). Al tiempo que reflexionan sobre esa experiencia real, adquieren un mayor conocimiento y comprensión, y cultivan su responsabilidad cívica y social (Puig et al., 2009, p.30).

El ApSU ha mostrado tener un impacto positivo en el aprendizaje académico, en el desempeño profesional, en el desarrollo personal y social (Chiva-Bartoll, SantosPastor, Martínez-Muñoz y Salvador-García, 2019; Rodríguez, 2014) (ver Tabla 1).

Sin embargo, el uso de una metodología activa no garantiza por sí misma que se consigan estos efectos, siendo imprescindible activar procedimientos y herramientas que acompañen los procesos formativos. En esta línea, el carácter transformador del ApSU favorece el desarrollo de un pensamiento crítico en el marco de la disciplina concreta (Educación Física), que es necesario para que convierta la experiencia en aprendizajes (Boud, 2001). En el ApSU, los procesos reflexivos incrementan el aprendizaje y las competencias del alumnado, logrando mejores niveles de éxito, así como un cambio de mirada hacia el otro, posibilitando "un incremento radical con la alteridad" (Chiva y Gil, 2018, p. 46). La reflexión ayuda a pensar la realidad sobre la que se interviene, al tiempo que la ilumina y resuelve problemas o interrogantes, 
aportando sentido personal y social al aprendizaje (Páez y Puig, 2013, p. 15). La opción de reflexionar sobre la realidad, permite revisarla de forma más profunda, ya que imprime sentido único a la experiencia y optimiza el aprendizaje (Kolb, 1984). Igualmente, la reflexión es el vínculo idóneo entre necesidades, servicio y aprendizaje (Brockbank y McGill, 2002; Martín, 2009). Sanders, Vans y McGeary (2015) ven en la reflexión la solución para evitar la explotación de los grupos desfavorecidos receptores del servicio, proponiendo relaciones reciprocas que desafían las ideas y prácticas establecidas, al tiempo que involucran al alumnado en procesos críticos, claves para un servicio socialmente justo.

Tabla 1. Efectos del ApSU

\begin{tabular}{|l|l|}
\hline \multicolumn{1}{|c|}{ Dimensiones } & \multicolumn{1}{c|}{ Efectos del ApsU } \\
\hline Académico & $\begin{array}{l}\text { Capacidad para aplicar lo aprendido en un mundo real (Domínguez, Me- } \\
\text { dina y López, 2018; León, Martínez y Santos, 2018), en la mejora de su } \\
\text { comprensión, en la resolución de problemas, en su pensamiento crítico } \\
\text { y en el desarrollo cognitivo, así como en la mejora para comprender la } \\
\text { complejidad (Eyler y Giles, 1999; Meaney et al.; 2008). }\end{array}$ \\
\hline Profesional & $\begin{array}{l}\text { Mejoran las relaciones con la comunidad, un mayor aprendizaje académi- } \\
\text { co (Martí, Corbatón y Serret, 2018), desarrollo de habilidades de liderazgo } \\
\text { y eficacia personal que pueden conducir a una mayor oportunidad (Celio, } \\
\text { Durlak y Dymnicki, 2011; Galvan, Meaney y Gray, 2018). }\end{array}$ \\
\hline Interpersonal & $\begin{array}{l}\text { Se adquiere mayor sentido de la eficacia en el desempeño, un aprendizaje } \\
\text { moral consciente y una mejora de la identidad, ayuda a que se aprenda } \\
\text { a trabajar con otras personas y a adquirir habilidades comunicativas y de } \\
\text { liderazgo (Melchior, 1999; Batlle, 2011; Rodríguez, 2014; Santos, Sotelino } \\
\text { y Lorenzo, 2015). }\end{array}$ \\
\hline Social & $\begin{array}{l}\text { Se genera un mayor entendimiento intercultural, se rompen estereotipos y } \\
\text { se adquiere una mayor responsabilidad social y ciudadana (Aramburuza- } \\
\text { bala, 2015; Lorente y Martos, 2018). }\end{array}$ \\
\hline
\end{tabular}

La reflexión es un elemento clave y tiene un papel crítico fundamental para que el ApSU adquiera sentido en la formación inicial y en la realidad social en la que se interviene, precisando el uso de herramientas que ayuden a canalizar "el análisis de la experiencia con la transformación de la mirada a través de nuevas lentes que ayudan a entender mejor lo vivido" (Páez y Puig, 2013, p. 29), además de un puente entre el aprendizaje y el servicio. Estas reflexiones deben ser orientadas mediante actividades útiles que ayudan al estudiante a evaluar su experiencia, considerar su valor y transformarlo en conocimiento para aplicarlo a su profesión y a la vida cotidiana como ciudadanos.

Al mismo tiempo, los procesos reflexivos deben ajustarse a las particularidades de los proyectos de ApSU y considerar los dinamismos que condicionan su desarrollo. Así, se distingue entre los dinamismos básicos, que contemplan las necesidades sociales, el servicio, el sentido social del servicio y los aprendizajes; y los 
dinamismos pedagógicos, donde situamos la participación, el trabajo en grupo, la reflexión, el reconocimiento y la evaluación (Ruiz-Corbella y García-Gutiérrez, 2019, p.141). Además, estos procesos formativos afectan a tres ámbitos: la experiencia personal, el escenario profesional y la formación en la práctica (Rubio y Escofet, 2017, p. 84).

Por tanto, desde estas consideraciones, tal y como han apuntado diferentes estudios (Chiva-Bartoll, Gil-Gómez y Zorrilla, 2019; Maravé, Gil y Chiva-Bartoll, 2019), el ApSU conecta con la experiencia personal del alumnado, implica los aprendizajes adquiridos tanto en la formación inicial como a lo largo de la vida y le preparan para actuar en un contexto concreto (aplicación práctica), vinculado con el ejercicio de la profesión (escenario profesional).

Pese a la proliferación de estudios realizados en los últimos años sobre el ApS (Chiva-Bartoll, et al., 2019), son escasos los que analizan los procesos reflexivos. Con el fin de dar respuesta a esta carencia y en consonancia con los planteamientos de otras investigaciones previas (Asghar y Rowe, 2016; Brown y Schmidt, 2016; Dubinsku, 2006; Lamoneda, Carter-Thuillier y López-Pastor, 2019; Mayor, 2019, 2020; Sanders, Vans y McGeary, 2015; Smit, 2017), analizamos los procesos reflexivos del alumnado de proyectos de ApSU, para comprender el valor que puede tener en su formación. En concreto, trataremos de identificar qué tipos de reflexiones realiza el alumnado y cómo percibe que influyen en su desarrollo personal, en su formación académica, y/o en la transferencia a su futura práctica profesional. Nuestra mayor aportación es valorar los efectos de los procesos reflexivos en contextos formativos de Actividad Física y Deporte (AFD).

\section{Método}

\subsection{Contextualización y participantes}

El estudio se desarrolló durante los cursos académicos 2018/19 y 2019/20, en los proyectos de ApSU-AFD que se realizaron desde la asignatura de Actividades Físico-Deportivas en el Medio Natural (AFDMN), impartida en $2^{\circ}$ curso del Grado en Ciencias de la Actividad Física y el Deporte, en la Universidad Autónoma de Madrid. En total participaron 25 estudiantes de Grado (6 grupos de 4-5 personas). Se realizaron cinco proyectos de ApSU-AFD con colectivos diferentes: mujeres en riesgo ${ }^{2}$, jóvenes en riesgo ${ }^{3}$ y jóvenes con discapacidad intelectual ${ }^{4}$, un total de 88 receptores del servicio (ver Tabla 2).

2. Mujeres sin hogar en situación de desventaja que participan en los programas de la Fundación Faciam.

3. Jóvenes de entre 15 y 23 años en riesgo de exclusión que participan en un programa de tiempo joven de la Fundación Tomillo.

4. Jóvenes de entre 18 y 23 años que presentan discapacidad intelectual leve o moderada y, en casos concretos, discapacidad motora, aunque con un alto grado de autonomía funcional, que participan en un programa formativo para la inclusión laboral de la Cátedra Prodis-UAM, denominado Promentor, distribuidos en dos cursos. 
Tabla 2. Distribución de participantes, proyectos y receptores del servicio. Fuente: elaboración propia

\begin{tabular}{|c|c|c|c|c|}
\hline Curso & $\begin{array}{c}\text { Grupo/no } \\
\text { estudiantes }\end{array}$ & $\begin{array}{c}\text { Sexo } \\
\text { (chico-chica) }\end{array}$ & Colectivo receptor & $\begin{array}{l}N^{\circ} \\
\text { receptores }\end{array}$ \\
\hline \multirow{4}{*}{ 2018/2019 } & $\mathrm{G} 1 / 5$ & $5-0$ & $\begin{array}{l}\text { Jóvenes con discapacidad intelectual } \\
1^{\circ} \text { curso }\end{array}$ & 17 \\
\hline & $\mathrm{G} 2 / 5$ & $2-3$ & $\begin{array}{l}\text { Jóvenes con discapacidad intelectual } \\
2^{\circ} \text { curso }\end{array}$ & 16 \\
\hline & $\mathrm{G} 3 / 4$ & $3-2$ & Mujeres sin hogar & 16 \\
\hline & $\mathrm{G} 4 / 5$ & $3-2$ & Jóvenes en riesgo & 9 \\
\hline \multirow{2}{*}{ 2019/2020 } & G5/3 & $2-1$ & $\begin{array}{l}\text { Jóvenes con discapacidad intelectual } \\
1^{\circ} \text { curso }\end{array}$ & 15 \\
\hline & G6/3 & $1-2$ & $\begin{array}{l}\text { Jóvenes con discapacidad intelectual } \\
2^{\circ} \mathrm{Curso}\end{array}$ & 15 \\
\hline
\end{tabular}

\subsection{Descripción del programa}

El proyecto consistió en un trabajo en grupo de carácter voluntario, implementando diferentes acciones de enseñanza y aprendizaje vinculadas a los contenidos específicos de las AFDMN con los receptores. El proceso constó de diferentes fases: (1) preparación (diagnóstico-diseño), (2) acción-intervención, (3) evaluación-valoración de la acción y (4) reflexión y replanteamiento de la acción. Los objetivos del servicio se orientaron a aumentar la competencia motriz en contacto con la naturaleza de los colectivos receptores, desarrollando AFDMN vinculadas a valores medioambientales (concienciación, respeto y cuidado). Los objetivos de aprendizaje se centraron en la aplicación de los contenidos de la asignatura a una situación real, mediante la implementación de una propuesta concreta de actividades (senderismo, orientación, marcha nórdica, etc.), adaptada a las necesidades de los receptores. En este proyecto se llevó a cabo una evaluación formativa, mediante una serie de procedimientos y de herramientas para asesorar y orientar al alumnado, informándole de las necesidades de reconstrucción de las tareas, ayudándole a reflexionar sobre sus fortalezas y debilidades (Tabla 3).

Tabla 3. Procedimientos de evaluación formativa en proyecto de ApSU-AFD. Fuente: elaboración propia

\begin{tabular}{|l|c|c|}
\hline \multicolumn{1}{|c|}{ Instrumentos de evaluación } & Carácter & Momento \\
\hline Cuestionarios reflexivos & Individual/grupal & Durante \\
\hline Portafolio & Individual & Durante \\
\hline Diario de aprendizajes & Individual & Durante \\
\hline Entrevista de evaluación final & Grupal & Final \\
\hline
\end{tabular}


En los diferentes instrumentos de evaluación se propusieron procesos reflexivos acordes con la naturaleza de cada una de las fases y acciones del proyecto, constituyéndose como una clave fundamental en los procesos formativos (Tabla 4). Con el fin de canalizar estos procesos reflexivos se han diseñado unos cuestionarios on-line que se aplicaron por conveniencia en momentos específicos del proyecto (diferentes fases y acciones vinculadas con el seguimiento y asesoramiento del proyecto). El objetivo fundamental era conseguir repensar los procesos vividos de manera inmediata, evitando que trascurriera mucho tiempo y se olvidasen aspectos relevantes, haciendo más consciente y significativo el aprendizaje.

Tabla 4. Cuestionarios para la reflexión del ApS en AFD. Fuente: elaboración propia

\begin{tabular}{|c|c|c|}
\hline $\begin{array}{c}\text { Tipo de } \\
\text { información }\end{array}$ & Ítems & Fase \\
\hline 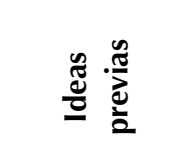 & $\begin{array}{l}\text { ¿Por qué has elegido hacer un proyecto de ApS? } \\
\text { ¿Cuáles son los compromisos personales que adquieres en el proyecto? } \\
\text { ¿Cuáles son tus expectativas ante el proyecto? } \\
\text { ¿Qué crees que puedas aportar? }\end{array}$ & इ. \\
\hline 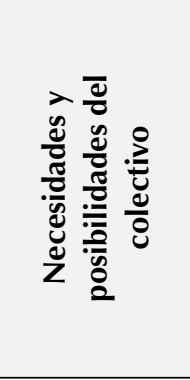 & $\begin{array}{l}\text { Características principales del colectivo } \\
\text { Posibilidades que tiene el colectivo para participar en el proyecto } \\
\text { Necesidades que presenta el colectivo } \\
\text { ¿Cuáles podrían ser prioritarias? } \\
\text { Objetivos del servicio que os planteáis cubrir en el proyecto } \\
\text { Acciones que podríais desarrollar en el proyecto } \\
\text { Título de vuestro proyecto ApSU-AFMN } \\
\text { ¿Qué necesitáis para ponerlo en práctica? } \\
\text { ¿Qué dificultades os surgen para desarrollar el proyecto? }\end{array}$ & 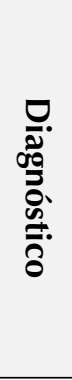 \\
\hline 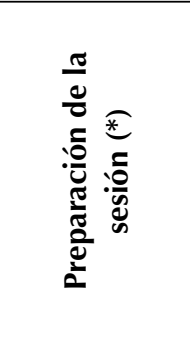 & $\begin{array}{l}\text { ¿Has tenido en cuenta las necesidades del colectivo para hacer el } \\
\text { diseño del proyecto de ApSU-AFDMN? } \\
\text { ¿Cómo lo has hecho? } \\
\text { ¿Qué has tenido en cuenta? } \\
\text { ¿Qué ha supuesto para ti preparar la intervención? } \\
\text { ¿Qué dificultades te han surgido en esta fase? } \\
\text { ¿Cómo las has solventado? } \\
\text { ¿Qué has aprendido con esta reflexión? }\end{array}$ & D. \\
\hline 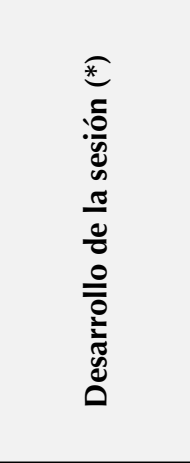 & $\begin{array}{l}\text { No de intervención del proyecto } \\
\text { Rol que has asumido } \\
\text { ¿En qué grado consideras que habéis conseguido los objetivos } \\
\text { propuestos? } \\
\text { ¿Qué dificultades has tenido individualmente? } \\
\text { ¿Qué dificultades han surgido en el grupo? } \\
\text { ¿Qué has aprendido? } \\
\text { ¿Qué cambios realizarías? } \\
\text { ¿Qué crees que has aportado al colectivo? } \\
\text { Valora la calidad del servicio realizado }(1 \text { al } 5) \\
\text { ¿Qué valoración darías a tu implicación en esta sesión? }(1 \text { al 5) }\end{array}$ & $\begin{array}{l}\overline{\overline{3}} \\
\frac{0}{0} \\
\frac{0}{3} \\
\frac{0}{2} \\
\frac{0}{2} \\
\frac{0}{0} \\
\frac{0}{3}\end{array}$ \\
\hline
\end{tabular}




\begin{tabular}{|c|c|c|}
\hline $\begin{array}{c}\text { Tipo de } \\
\text { información }\end{array}$ & Ítems & Fase \\
\hline 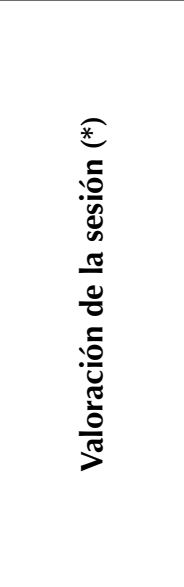 & $\begin{array}{l}\text { Colectivo con el que has desarrollado el proyecto } \\
\text { Número de intervenciones realizadas } \\
\text { ¿Te ha parecido adecuado el número de sesiones dedicado al desarrollo } \\
\text { del proyecto? Justifica tu respuesta } \\
\text { ¿Qué es lo que más te ha ayudado a desarrollar el proyecto? } \\
\text { ¿Qué has aprendido? } \\
\text { ¿Qué dificultades has tenido? } \\
\text { ¿Qué beneficios aporta a tu formación profesional y personal? } \\
\text { ¿Qué consideras que has aportado a los receptores del servicio? } \\
\text { ¿Qué ha sido lo más valioso de este proyecto? } \\
\text { Valora la aportación del proyecto de ApS a tu formación profesional } \\
\text { (1 al 5) } \\
\text { Valora la aportación del proyecto de ApS a tu formación personal (1 } \\
\text { al 5) }\end{array}$ & 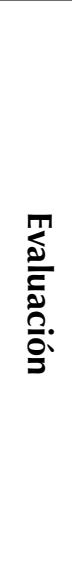 \\
\hline 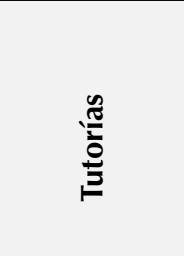 & $\begin{array}{l}\text { Motivo de la tutoría } \\
\text { Tiempo aproximado que ha durado } \\
\text { Temas tratados en la tutoría } \\
\text { ¿Qué has aportado a la mejora del proyecto? } \\
\text { ¿Qué has aprendido? } \\
\text { ¿Qué mejorarías? }\end{array}$ & 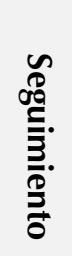 \\
\hline
\end{tabular}

(*) Un cuestionario por cada propuesta de sesión.

Los cuestionarios se enviaron al alumnado por correo electrónico al finalizar cada fase. Se les solicitaba completarlos en un plazo corto para recordar lo vivido, evitando así la pérdida de información significativa.

\subsection{Técnicas de recogida}

La información se ha recogido mediante los cuestionarios reflexivos señalados anteriormente, que aportaban una perspectiva tanto personal como grupal sobre los aprendizajes.

\subsection{Análisis de la información}

Se ha empleado el análisis de contenido desde el enfoque cualitativo como "conjunto de técnicas de análisis de las comunicaciones utilizando procedimientos sistemáticos y objetivos de descripción del contenido de los mensajes" (Bardin, 1996, p. 29). Se trata de una técnica que permite la reelaboración y reducción de los datos que nos ayuda a entresacar el contenido manifiesto y latente de los datos analizados, agruparlos en unidades de significado, así como establecer relaciones entre los temas tratados (Mayring, 2000). 
Se han establecido unas categorías previas, elaborando una matriz con la que poder obtener una representación de los datos, reducirlos, transformarlos y sacar conclusiones valiosas de la realidad objeto de estudio (Miles y Huberman, 1984). Estas categorías de análisis se han extraído de la bibliografía, así como del análisis previo de las unidades de análisis de los datos recopilados. Igualmente, apoyándonos en la propuesta realizada por Rubio y Escofet (2017, p. 20), consideramos que las reflexiones realizadas por el alumnado se centran en tres ámbitos: la experiencia personal, el escenario profesional y la formación en la práctica, relacionándolos con los dinamismos básicos y pedagógicos de los proyectos de ApSU-AFD (Tabla 5).

Tabla 5. Componentes de análisis de las reflexiones. Dimensiones y ámbitos. Fuente: elaboración propia

\begin{tabular}{|l|l|l|l|}
\hline \multicolumn{1}{|c|}{ ÁMBITOS } & \multicolumn{1}{|c|}{ COMPONENTES } & \multicolumn{2}{c|}{ DIMENSIONES } \\
\hline \multirow{2}{*}{ Experiencia personal } & \multirow{2}{*}{ Aprendizaje } & $\begin{array}{l}\text { Competencias espe- } \\
\text { cíficas }\end{array}$ & $\begin{array}{l}\text { Competencias trans- } \\
\text { versales }\end{array}$ \\
\cline { 3 - 4 } & & Necesidades & Posibilidades \\
\hline \multirow{2}{*}{ Formación inicial } & \multirow{2}{*}{ Servicio } & Sentido & Participación \\
\cline { 3 - 5 } & & Trabajo en equipo & Evaluación \\
\hline Escenario profesional & Aprendizaje-Servicio & Reconocimiento & \multicolumn{2}{|c|}{} \\
\hline
\end{tabular}

Una vez realizado el análisis del contenido, atendiendo a las categorías propuestas, se hizo un recuento cuantitativo de los datos, obteniendo las frecuencias de las reflexiones de los cuestionarios según dichas categorías.

La información recopilada ha sido sometida al consentimiento de los participantes para poder emplearla. Los criterios de rigor utilizados han sido: la triangulación de técnicas, la transferibilidad, la dependencia y la confirmabilidad.

\section{Resultados Y DISCUSIÓN}

Los datos recopilados nos permiten analizar y comprender el valor de la reflexión en esta propuesta formativa. Un primer acercamiento a los procesos reflexivos en las diferentes etapas del proyecto permite apreciar las frecuencias de las reflexiones en función de los ámbitos, los componentes y las dimensiones presentes en los proyectos de ApSU-AFD (ver Tabla 2).

Como apunte general (Tabla 6), podemos indicar que, de los 465 registros considerados válidos, el 14.2\% hacen referencia al ámbito de experiencia personal, el $79.6 \%$ a la formación inicial y el $6.24 \%$ al escenario profesional. La dimensión más destacada es la relativa a las competencias específicas (46.89\%) y transversales (35.75\%), seguida del sentido del servicio (8.18\%). Como reflexiones generadas en menor medida, recogemos el trabajo en equipo $(6.31 \%)$ y las posibilidades del servicio $(4.95 \%)$. Entre las menos valoradas están las reflexiones sobre la evaluación del proyecto $(0.87 \%)$, con escasa consideración en ningún ámbito ni componente del ApSU-AFD. 
Tabla 6. Porcentajes sobre reflexiones en ApSU-AFD por ámbitos, componentes y dimensiones. Fuente: elaboración propia

\begin{tabular}{|l|c|c|c|}
\hline \multirow{4}{*}{} & $\begin{array}{c}\text { Experiencia } \\
\text { personal }\end{array}$ & $\begin{array}{c}\text { Formación } \\
\text { inicial }\end{array}$ & $\begin{array}{c}\text { Escenario } \\
\text { profesional }\end{array}$ \\
\cline { 2 - 4 } & 14.2 & 79.6 & 6.2 \\
\hline & COMPONENTES \\
\hline & Aprendizaje & Servicio & $\begin{array}{c}\text { Aprendizaje- } \\
\text { Servicio }\end{array}$ \\
\cline { 2 - 4 } & 76.3 & 17 & 6.7 \\
\hline DIMENSIONES \\
\hline Competencias específicas & - & 42.37 & 4.52 \\
\hline Competencias transversales & 3.23 & 25.16 & 7.36 \\
\hline Sentido del servicio & 5.81 & 2.37 & 0.22 \\
\hline Posibilidades del servicio & 2.58 & 2.37 & - \\
\hline Trabajo en grupo & 1.05 & 5.26 & - \\
\hline Evaluación & 0.22 & 0.65 & - \\
\hline
\end{tabular}

En cuanto a las reflexiones generadas atendiendo a las fases de los proyectos de ApSU-AFD (ver Tabla 5), encontramos que gran parte se dirigen al aprendizaje (adquisición de competencias específicas y transversales), destacando sobre todo en la fase de intervención (87.25\%) y en la tutoría con los grupos de trabajo $(82.94 \%)$, siendo la de diagnóstico la que menor incidencia presenta. En relación con el servicio que se realiza, destacan las reflexiones del diagnóstico de necesidades de los colectivos (40\%) que, sin embargo, contrasta con la tutoría como la menos valorada (6.25\%). Por su parte, el componente de Aprendizaje-Servicio es el que en su conjunto adquiere menos consideración en las reflexiones, siendo la evaluación final (13.67\%) y el diagnóstico (12\%) los que mayor interés plantean (Tabla 7 ).

La valoración global que establecen sobre la contribución del proyecto a su formación profesional es de 5 (sobre 5) para el 80\% del alumnado, mientras que el $67 \%$ valora con 4 (sobre 5) la aportación que ha tenido en su formación personal.

Tabla 7. Porcentaje en las reflexiones en el ApS por fases y componentes. Fuente: elaboración propia

\begin{tabular}{|l|c|c|c|}
\hline \multicolumn{1}{|c|}{ FASE } & APRENDIZAJE & SERVICIO & APRENDIZAJE-SERVICIO \\
\hline Diagnóstico & 48 & 40 & 12 \\
\hline Diseño & 61.29 & 29.02 & 9.69 \\
\hline Práctica & 87.25 & 11.76 & 0.98 \\
\hline Tutorías & 82.94 & 6.25 & 7.81 \\
\hline Evaluación Final & 58.94 & 27.38 & 13.67 \\
\hline
\end{tabular}


Tabla 8. Unidades de análisis de las reflexiones. Fuente: elaboración propia

\begin{tabular}{|c|c|c|}
\hline \multicolumn{3}{|c|}{ DIMENSIONES } \\
\hline [CE] Competencias específicas & [P] Posibilidades & [PA] Participación \\
\hline [CT] Competencias transversales & [Sse] Sentido-Servicio & [RE] Replanteamiento \\
\hline [N] Necesidades & [TE] Trabajo en equipo & [EV] Evaluación \\
\hline \multicolumn{3}{|c|}{ FASES } \\
\hline [DIAG] Diagnóstico & [Pr] Práctica & [EF] Evaluación Final \\
\hline [DIS] Diseño & [T] Tutorías & \\
\hline \multicolumn{3}{|c|}{ COMPONENTES } \\
\hline [A] Aprendizaje & [S] Servicio & [AS] Aprendizaje-Servicio \\
\hline
\end{tabular}

Los resultados obtenidos muestran que en los proyectos de ApSU-AFD los procesos reflexivos son muy útiles para hacer consciente al alumnado de lo que aprende, de lo que necesita aprender, de lo que hace y del sentido de lo que hace, y de lo que aprende, como se ha constatado en estudios recientes (Pagalagar, 2018). Así, podemos comprender que el ámbito que está más presente en la reflexión del alumnado es el relacionado con los efectos que tiene su participación e implicación en su formación académica, idea que se ha puesto de manifiesto en otros trabajos (Capraro, Capraro y Helfeldt, 2010). Lo que no parece coincidir con las consideraciones expuestas en otras investigaciones es la escasa aportación de estas propuestas a su experiencia personal (Capella-Peris, Gil-Gómez y Chiva-Bartoll, 2019) y al desarrollo de su profesión (Reyes y Martínez, 2019). Si analizamos con detalle la consideración que hacen de los distintos componentes que integran el proyecto, observamos que el aprendizaje predomina sobre el servicio, y éste por encima del aprendizaje-servicio, aunque también es relativo a la fase del proceso. Esto es un indicador de que el alumnado valora este tipo de proyectos como una propuesta metodológica novedosa para trabajar los contenidos académicos y ponerlos en práctica, más que como un enfoque formativo integral. Este hallazgo coincide en parte con lo que propone el estudio de Maravé, Gil y Chiva-Bartoll (2019).

En relación con las dimensiones de los proyectos, observamos que sus reflexiones se orientan hacia la adquisición de las competencias, valorando especialmente las relacionadas con el contenido curricular (AFDMN), siendo las transversales menos apreciadas, aunque están vinculadas con los receptores. Además, conviene estimar las reflexiones en función de las fases del proyecto, siendo sobre la intervención y las tutorías donde más incidencia tiene (Tabla 9). 
Tabla 9. Análisis de las reflexiones recogidas en los cuestionarios

\begin{tabular}{|c|c|c|c|c|}
\hline Reflexiones & Ámbito & Fase & Componente & Dimensión \\
\hline $\begin{array}{l}\text { Como dirigir una clase, controlar que el } \\
\text { alumnado desempeñe o realice las tareas } \\
\text { de forma correcta para que se cumplan } \\
\text { los objetivos propuestos y a empatizar con } \\
\text { este colectivo (Cuest-RE-PRA, AL.5) }\end{array}$ & FI & Pr & A & CE/CT \\
\hline $\begin{array}{l}\text { Anticiparnos ante las adversidades me- } \\
\text { teorológicas, diseñar y planificar sesiones } \\
\text { y saber a que nos exponemos, teniendo } \\
\text { siempre en cuenta lo que queremos ense- } \\
\text { ñar (Cuest-RE-TUT, AL.6) }\end{array}$ & FI & TUT & A & CE \\
\hline
\end{tabular}

Se puede apreciar que, pese a que el aprendizaje se sitúa en el centro de atención de sus reflexiones, son consientes de que los receptores del servicio aportan un significado único a su aprendizaje (Tabla 10).

Tabla 10. Análisis de las reflexiones recogidas en los cuestionarios

\begin{tabular}{|c|c|c|c|c|}
\hline Reflexiones & Ámbito & Fase & Componente & Dimensión \\
\hline $\begin{array}{l}\text { El descubrimiento de nuevas formas de ha- } \\
\text { cer actividad física de manera lúdica y en } \\
\text { un entorno llamativo y diferente como es el } \\
\text { medio natural. Además, se les instó a cui- } \\
\text { darlo y protegerlo (Cuest-RE-PRA, AL.19) }\end{array}$ & FI & Pr & S & Sse \\
\hline $\begin{array}{l}\text { He aprendido bastante, sobre todo a es- } \\
\text { tructurar un proyecto de AFDMN y a saber } \\
\text { gestionarlo (Cuest-RE-TUT, AL.5) }\end{array}$ & FI & TUT & A & EF \\
\hline
\end{tabular}

Se comprueba que involucrarse en los proyectos de ApSU-AFD produce sentimientos especiales hacia el colectivo con el que se trabaja, valorándose como algo prioritario de la experiencia, provocando un cambio de mirada hacia el otro (Chiva y Gil, 2018, p. 46). De alguna forma, parece que la desventaja que presentan los colectivos receptores sensibiliza al alumnado sobre su realidad y le hace concienciarse de la importancia de participar en este proyecto con responsabilidad, aportando lo mejor de sus aprendizajes (Tabla 11).

De hecho, sienten motivación por lo que pueden aportar al colectivo receptor para mejorar su bienestar. Se muestran sensibles para hacer un servicio adecuado, aunque ello les exija preparar, consultar o estudiar con detalle cómo hacerlo. De acuerdo con Brockbank y McGill (2002), se aprecia que los conocimientos que necesitan están vinculados con la necesidad de adaptar los aprendizajes académicos a la práctica real y a las limitaciones del colectivo, uniendo necesidades, servicio 
y aprendizaje. Esta investigación pone de manifiesto la importancia de hacer una buena selección de tareas, valorando la importancia de generar prácticas verdaderamente inclusivas (Tabla 12).

Tabla 11. Análisis de las reflexiones recogidas en los cuestionarios

\begin{tabular}{|c|c|c|c|c|}
\hline Reflexiones & Ámbito & Fase & Componente & Dimensión \\
\hline $\begin{array}{l}\text { Me ha supuesto una satisfacción plena, ya } \\
\text { que era consciente que el aprendizaje de } \\
\text { un grupo de personas tan maravillosa esta- } \\
\text { ba bajo mi cargo (Cuest-RE-DIS, AL.1) }\end{array}$ & EP & DIS & S & Sse \\
\hline $\begin{array}{l}\text { Personalmente, creo que lo que aporto no } \\
\text { se puede expresar mediante palabras. Sólo } \\
\text { puedo decir que me hace muy feliz ver que } \\
\text { ellos están contentos y satisfechos a través } \\
\text { de sesiones dirigidas por nosotros (Cuest- } \\
\text { RE-PRA, AL.3) }\end{array}$ & EP & Pr & S & Sse \\
\hline
\end{tabular}

Tabla 12. Análisis de las reflexiones recogidas en los cuestionarios

\begin{tabular}{|c|c|c|c|c|}
\hline Reflexiones & Ámbito & Fase & Componente & Dimensión \\
\hline $\begin{array}{l}\text { Dependiendo de cada una de las necesi- } \\
\text { dades de la clase, hemos establecido unas } \\
\text { necesidades básicas comunes para todos } \\
\text { (Cuest-RE-DIS, AL.1) }\end{array}$ & FI & DIS & S & $\mathrm{N}$ \\
\hline $\begin{array}{l}\text { He aprendido que es importante prestar } \\
\text { más atención a este colectivo, ya que ellos } \\
\text { valoran mucho nuestro trabajo y están } \\
\text { siempre muy agradecidos por todo lo que } \\
\text { hacemos por ellos (Cuest-RE-EV, AL.3) }\end{array}$ & FI & EV & S & EF \\
\hline
\end{tabular}

Así, la seguridad de la práctica se percibe como una preocupación fundamental para que puedan participar en condiciones que no suponga ningún riesgo para el servicio (Tabla 13). Esta reflexión supone tener en cuenta las acciones, su manera de diseñarlas y ponerlas en práctica. De hecho, se constata que reflexionan sobre los aprendizajes que adquieren vinculándolos con los contenidos de la asignatura (AFDMN) y que se ve reforzado por buscar alternativas diferentes de aplicación para la adaptación al servicio del colectivo con el que trabajan. Además, consideran prioritario adaptarse a la heterogeneidad del grupo receptor y a sus necesidades/posibilidades/limitaciones, algo que valoran como positivo para su formación como docentes, rompiendo estereotipos y mejorando la responsabilidad social y ciudadana, tal y como apuntaban Lorente y Martos (2018). 
Tabla 13. Análisis de las reflexiones recogidas en los cuestionarios

\begin{tabular}{|l|c|c|c|c|}
\hline \multicolumn{1}{|c|}{ Reflexiones } & Ámbito & Fase & Componente & Dimensión \\
\hline $\begin{array}{l}\text { He tenido en cuenta el grupo con el que } \\
\text { trabajábamos, sus limitaciones, su destre- } \\
\text { za motriz y agentes externos (Cuest-RE- } \\
\text { DIS, AL.5) }\end{array}$ & FI & DIS & S & N \\
\hline $\begin{array}{l}\text { Y así poder realizar tareas más accesi- } \\
\text { bles para ellos en cuanto a nivel de eje- } \\
\text { cución o toma de decisiones (Cuest-RE- } \\
\text { DIS, AL.1) }\end{array}$ & FI & DIS & A & CE \\
\hline
\end{tabular}

Por otra parte, entienden que es muy importante dominar el conocimiento del contenido de la materia y tener presente el conocimiento pedagógico del contenido o su aplicación a la práctica (Tabla 14). Aspectos como la estructura de la intervención, control de los tiempos, número de participantes, comunicación eficaz, organización de los materiales y espacios, duración de las tareas, control del grupo, resolución de conflictos, motivación, participación, etc., permiten mejorar su capacidad para aplicar lo aprendido al mundo real (Domínguez, Medina y López, 2018).

Tabla 14. Análisis de las reflexiones recogidas en los cuestionarios

\begin{tabular}{|c|c|c|c|c|}
\hline Reflexiones & Ámbito & Fase & Componente & Dimensión \\
\hline $\begin{array}{l}\text { La única dificultad es controlar el tiempo, } \\
\text { pero eso con un pelín más de experiencia } \\
\text { saldrá bien (Cuest-RE-PRA, AL.5) }\end{array}$ & FI & $\operatorname{Pr}$ & A & RE \\
\hline $\begin{array}{l}\text { La falta de tiempo para preparar la activi- } \\
\text { dad y tener todo listo antes de que viniera } \\
\text { el alumnado (Cuest-RE-PRA, AL.11) }\end{array}$ & FI & $\operatorname{Pr}$ & A & EF \\
\hline
\end{tabular}

La falta de conocimiento didáctico se refleja en sus reflexiones de manera constante, dejando entrever que es una de sus principales debilidades (Tabla 15). En sus reflexiones muestran de forma insistente cierta inseguridad para desarrollar la práctica, ser competentes para aplicar los conocimientos adquiridos en su formación inicial y asegurar el éxito en la aplicación de la sesión. De igual forma, manifiestan tener en cuenta sus limitaciones y muestran la importancia de tener que seguir formándose. Sin duda, como anunciaba Puig et al. (2009), estas experiencias formativas ayudan al estudiante adquirir mayor conocimiento y comprensión de la realidad. 
Tabla 15. Análisis de las reflexiones recogidas en los cuestionarios

\begin{tabular}{|l|c|c|c|c|}
\hline \multicolumn{1}{|c|}{ Reflexiones } & Ámbito & Fase & Componente & Dimensión \\
\hline $\begin{array}{l}\text { Poder seguir aprendiendo sobre este co- } \\
\text { lectivo (Cuest-RE-DIAG, AL.1) }\end{array}$ & EP & DIAG & A & P \\
\hline $\begin{array}{l}\text { El contacto con el colectivo y el corres- } \\
\text { pondiente desarrollo a nivel personal } \\
\text { (Cuest-RE-EV, AL.7) }\end{array}$ & EP & EV & S & Sse \\
\hline $\begin{array}{l}\text { Mejorar como docente (Cuest-RE-EV, } \\
\text { AL.8) }\end{array}$ & EP & EV & A & CE \\
\hline
\end{tabular}

Igualmente, hemos recogido algunas reflexiones en las que ponen en valor aqueIlo que pueden aportar al servicio, sintiendo cierta responsabilidad por hacerlo con calidad (Tabla 16). Piensan que su aportación al colectivo es importante, teniendo un impacto positivo en su desarrollo personal y social, tal y como señalan ChivaBartoll et al. (2019). Además de buscar en los receptores aprendizajes concretos relacionados con la actividad física y el deporte, son sensibles a proporcionar valores relacionados con la práctica y favorecer su bienestar personal y social. De hecho, cuando se trata de colectivos jóvenes, les preocupa el aprendizaje motriz, mientras que cuando son adultos (como en el caso de las mujeres), optan por generar experiencias positivas en la práctica y favorecer la socialización con el grupo (Martí, Corbatón y Serret, 2018).

Tabla 16. Análisis de las reflexiones recogidas en los cuestionarios

\begin{tabular}{|l|c|c|c|c|}
\hline \multicolumn{1}{|c|}{ Reflexiones } & Ámbito & Fase & Componente & Dimensión \\
\hline $\begin{array}{l}\text { Felicidad y conocimientos de como cui- } \\
\text { dar el medio ambiente (Cuest-RE-PRA, } \\
\text { AL.25) }\end{array}$ & FI & $\operatorname{Pr}$ & $\mathrm{A}$ & Sse \\
\hline $\begin{array}{l}\text { Motivación y ganas por practicar depor- } \\
\text { te en un medio natural (Cuest-RE-PRA, } \\
\text { AL.27) }\end{array}$ & FI & $\operatorname{Pr}$ & $\mathrm{S}$ & Sse \\
\hline
\end{tabular}

Por otro lado, manifiestan en sus reflexiones las dificultades que tienen para llevar a cabo estos proyectos, comprendiendo que la falta de experiencia justifica ciertas debilidades de la aplicación práctica (Tabla 17). Incluso se sienten con baja cualificación cuando, por algún motivo, tienen que improvisar o tomar decisiones no programadas. Coincidiendo con otras investigaciones, también se percibe con claridad las debilidades que han tenido y les hace repensar-reconstruir aquello que tendrían que corregir para próximas acciones, mejorando la resolución de problemas, su pensamiento crítico y la comprensión de la complejidad de la realidad (Eyler y Giles, 1999; Santos, Martínez y Cañadas, 2020). 
Tabla 17. Análisis de las reflexiones recogidas en los cuestionarios

\begin{tabular}{|c|c|c|c|c|}
\hline Reflexiones & Ámbito & Fase & Componente & Dimensión \\
\hline $\begin{array}{l}\text { Además, gracias a realizar el proyecto en } \\
\text { esta asignatura, he podido reflexionar so- } \\
\text { bre lo perdidos que estamos a la hora de } \\
\text { realizar sesiones (Cuest-RE-DIS, AL.3) }\end{array}$ & FI & DIS & AS & EF \\
\hline $\begin{array}{l}\text { Algunas adaptaciones de última hora por el } \\
\text { tiempo o por el material y lo de controlar } \\
\text { el final de las tareas (Cuest-RE-PRA, AL.1) }\end{array}$ & FI & Pr & A & RF \\
\hline
\end{tabular}

De modo general, el alumnado valora la reflexión como un aspecto clave que les ayuda a orientar cada fase del proyecto y las acciones que tienen que realizar en cada una de ellas: tomar decisiones, saber qué necesitan y cómo tienen que hacerlo (Tabla 18).

Tabla 18. Análisis de las reflexiones recogidas en los cuestionarios

\begin{tabular}{|c|c|c|c|c|}
\hline Reflexiones & Ámbito & Fase & Componente & Dimensión \\
\hline $\begin{array}{l}\text { Con esta reflexión he aprendido que la } \\
\text { finalidad de cada sesión es clave para } \\
\text { posteriormente realizar una progresión } \\
\text { de tareas adecuadas en el medio natural } \\
\text { (Cuest-RE-DIS, AL.3) }\end{array}$ & FI & DIS & A & CE \\
\hline $\begin{array}{l}\text { Con la reflexión te das cuenta de lo que } \\
\text { verdaderamente has aprendido y el empe- } \\
\text { ño que le has puesto para la realización } \\
\text { (Cuest-RE-DIS, AL.1) }\end{array}$ & EP & DIS & A & EF \\
\hline
\end{tabular}

Además, pensar sobre la acción desarrollada, les ayuda a apreciar lo que van aprendido y lo que les ha supuesto esta experiencia en su formación inicial (Tabla 19).

Tabla 19. Análisis de las reflexiones recogidas en los cuestionarios

\begin{tabular}{|l|c|c|c|c|}
\hline \multicolumn{1}{|c|}{ Reflexiones } & Ámbito & Fase & Componente & Dimensión \\
\hline $\begin{array}{l}\text { Las experiencias vividas que favorecen mi } \\
\text { desarrollo personal y profesional (Cuest- } \\
\text { RE-EV, AL.10) }\end{array}$ & EP & EV & A & CE \\
\hline $\begin{array}{l}\text { Es algo que, si en un futuro tengo que dedi- } \\
\text { carme, no me importaría y además no sería } \\
\text { algo nuevo para mi (Cuest-RE-EV, AL.12) }\end{array}$ & FI & EV & A & CT \\
\hline
\end{tabular}

Las tutorías son elementos clave para resolver sus dudas, mejorar los proyectos y aprender, pero sobre todo les incita a ser conscientes de lo que van aprendiendo y les invita a reflexionar sobre lo que necesitan para seguir haciéndolo (Tabla 20). 
Tabla 20. Análisis de las reflexiones recogidas en los cuestionarios

\begin{tabular}{|c|c|c|c|c|}
\hline \multicolumn{1}{|c|}{ Reflexiones } & Ámbito & Fase & Componente & Dimensión \\
\hline $\begin{array}{l}\text { El poder ser asesorado por docentes y } \\
\text { compañeros que ya habían estado in- } \\
\text { volucrados en el proyecto de antemano } \\
\text { (Cuest-RE-EV, AL.8) }\end{array}$ & FI & EV & A & CE \\
\hline
\end{tabular}

Por otro lado, perciben que la ayuda en el grupo de trabajo es muy importante, tanto para poder tener apoyo para suplir la carencia de ciertos conocimientos técnicos o didácticos, como recibir consejos prácticos por la experiencia anterior desarrollada (Tabla 21). Así, las reflexiones apuntan a que el trabajo en equipo facilita el proceso, complementa la labor de asesoramiento del docente, mejora las relaciones interpersonales, las habilidades comunicativas y de liderazgo (Santos, Sotelino y Lorenzo, 2015).

Tabla 21. Análisis de las reflexiones recogidas en los cuestionarios

\begin{tabular}{|c|c|c|c|c|}
\hline Reflexiones & Ámbito & Fase & Componente & Dimensión \\
\hline $\begin{array}{l}\text { Hablando con mis compañeros que tienen } \\
\text { experiencia en trabajar con este colectivo } \\
\text { y asistir a tutorías (Cuest-RE-DIS, AL.5) }\end{array}$ & FI & DIS & AP & TG \\
\hline
\end{tabular}

Por último, en sus reflexiones muestran diferentes emociones o sentimientos como respuesta a situaciones que viven a lo largo del proceso (Tabla 22). En ocasiones, dicen que sienten estrés por diferentes circunstancias, ya sea por lo que tienen que preparar para desarrollar el proyecto (planificación y programación), como coordinarlo con el colectivo receptor y las entidades, o por compaginarlo con otras actividades académicas. Incluso a veces sienten miedo por no poder responder a las expectativas del proyecto, ya sean en relación con el aprendizaje o con el servicio.

Tabla 22. Análisis de las reflexiones recogidas en los cuestionarios

\begin{tabular}{|l|c|c|c|c|}
\hline \multicolumn{1}{|c|}{ Reflexiones } & Ámbito & Fase & Componente & Dimensión \\
\hline $\begin{array}{l}\text { El miedo a que no resulte cómo esperá- } \\
\text { bamos o equivocarme, que me pregunten } \\
\text { algo y no pueda responder (Cuest-RE-EV, } \\
\text { AL.2) }\end{array}$ & ExP & EV & A & CE \\
\hline $\begin{array}{l}\text { La incertidumbre de las personas que ven- } \\
\text { drían, sobre todo porque los ejercicios no } \\
\text { estaban adaptados para tantos alumnos. } \\
\begin{array}{l}\text { Pero creo que hemos sabido adaptarlo a } \\
\text { la perfección (Cuest-RE-PRA, AL.17) }\end{array}\end{array}$ & FI & Pr & A & CE/RE \\
\hline $\begin{array}{l}\text { Conseguir una inclusión social hacia este } \\
\text { colectivo (Cuest-RE-DIAG, AL.3) }\end{array}$ & ExP & DIAG & S & Sse \\
\hline
\end{tabular}


La gestión de las emociones del alumnado es un aprendizaje clave que se puede promover mediante los procesos reflexivos conscientes, orientados y revisados. El alumnado toma de conciencia de sí mismo en relación con los otros o con el entorno. Las reflexiones sobre sus emociones, como alertaban algunos autores (García y Sánchez, 2017), se vinculan con la capacidad para tener buenas relaciones con las personas, adoptar comportamientos apropiados y responsables para responder de forma adecuada al contexto.

\section{Conclusión}

El presente estudio ha puesto en valor el interés de la reflexión para mejorar los procesos de aprendizaje del alumnado que participa en los proyectos de ApSU-AFD. La reflexión es un puente para dar sentido al ApSU como metodología de enseñanza y como enfoque formativo, vinculando las experiencias formativas reales con las necesidades de los colectivos receptores. La reflexión favorece el desarrollo de un pensamiento crítico en el marco de la disciplina concreta y la transformación de la experiencia en aprendizajes. La reflexión evita que el servicio prestado tenga carácter asistencial o sea una actividad caritativa, para convertirla en una acción personal y formativa valiosa para la construcción de una ciudadanía activa.

En conclusión, la reflexión es una garantía para que el ApSU sea un instrumento para una formación universitaria comprometida con la justifica social.

\section{REFERENCIAS BIBLIOGRÁFICAS}

Aramburuzabala, P. (2015). Aprendizaje-Servicio: Una herramienta para educar desde y para la Justicia Social. Revista Internacional De Educación Para La Justicia Social, 2(2), 5-11.

Asghar, M. y Rowe, N. (2017). Reciprocity and critical reflection as the key to social justice in service learning: A case study. Innovations in Education and Teaching, 54, 117-125. https://doi.org/10.1080/14703297.2016.1273788

Bardin, L. (1996). Análisis de contenido. Madrid: Akal Ediciones.

Batlle, R. (2011). ¿De qué hablamos cuando hablamos de aprendizaje servicio? Crítica, 972, 49-54.

Boud, D. (2001). Using Reflective Writing to Enhance Reflective Practice. New Directions for adult and Continuing Education, 90, 9-17.

Brockbank, A. y McGill, I. (2002). Aprendizaje reflexivo en la Educación Superior. Madrid: Morata.

Brown, J. J. y Schmidt, N. A. (2016). Service-Learning in Undergraduate Nursing Education: Where is the Reflection? Journal of Professional Nursing, 32, 48-53.

Capella-Peris, C., Gil-Gómez, J. y Chiva-Bartoll, Ò. (2019). Innovative Analysis of Service-Learning Effects in Physical Education: A Mixed Methods Approach. Journal of Teaching in Physical Education, 39(1), 102-110. doi: https://doi.org/10.1123/ jtpe.2019-0030 
Capraro, M. M., Capraro, R. M. y Helfeldt, J. (2010). Do differing types of field experiences make a difference in teacher candidates' perceived level of competence? Teacher Education Quarterly, 37(1), 131-154.

Celio, C. I., Durlak, J. y Dymnicki, A. (2011). A Meta-Analysis of the Impact of Service Learning on Students. Journal of Experiential Education, 34(2), 164-181.

Chiva-Bartoll, Ò. y Gil Gómez, J. (Eds.) (2018). Aprendizaje-Servicio Universitario. Modelos de intervención e investigación en la formación inicial docente. Barcelona: Octaedro.

Chiva-Bartoll, O., Pallarés-Piquer, M. y Gil-Gómez, J. (2018). Aprendizaje-servicio y mejora de la Personalidad Eficaz en futuros docentes de Educación Física. Revista Complutense de Educación, 29(1), 181-197.

Chiva-Bartoll, O., Ruiz-Montero, P., Martín Moya, R., Pérez López, I., Giles Girela, J., García-Suárez, J. y Rivera-García, E. (2019). University Service-Learning in Physical Education and Sport Sciences: A systematic review. Revista Complutense De Educación, 30(4), 1147-1164. https://doi.org/10.5209/rced.60191

Chiva-Bartoll, Ò., Santos-Pastor, M. L., Martínez-Muñoz, L. F. y Salvador-García, C. (2019). Valoración del impacto del aprendizaje-servicio universitario en el ámbito de la actividad física y el deporte: una propuesta desde la teoría de stakeholders. Publicaciones, 49(4), 27-46. http://doi.org/10.30827/ publicaciones.v49i4

Domínguez, M. C., Medina, A. y López, E. (2018). Desarrollo de competencias en el primer curso de universidad: estudio de caso. Publicaciones, 48(1), 47-62. https:// doi.org/10.30827/publicaciones.v48i1.7325

Dubinsku, J. (2006). The Role of reflection in Service Learrning. Business Communication Quarterly, 69(3). http://doi.org/10.1177/108056990606900308

Eyler, J. y Giles, J. (1999). Where's the Learning in Service-Learning. San Francisco, CA: Jossey-Bass.

Fandos, M., Renta, A. I., Jiménez, J. M. y Gonzáles, A. P. (2017). Análisis sobre el aprendizaje y la aplicación de las competencias generales en el contexto laboral. Estrategias de colaboración entre la formación profesional. La universidad y la empresa. Educar, 53(2), 333-355.

Galvan, C., Meaney, K. y Gray, V. (2018). Examining the Reciprocal Nature of ServiceLearning for Underserved Students and Preservice Teachers. Journal of Teaching in Physical Education, 37(4), 363-372.

García, M. y Sánchez, L. (2017). El aprendizaje servicio y el desarrollo de las competencias emocionales en la formación inicial del profesorado. Contextos Educativos, 20, 127-145. http://doi.org/10.18172/con.2991

Kolb, D. A. (1984). Experiential learning: Experience as the source of learning and development. New Jersey: Prentice Hall. 
Lamoneda, J., Carter-Thuillier, B. y López-Pastor, V. M. (2019). Efectos de un programa de aprendizaje servicio para el desarrollo de prosocialidad y actitudes positivas hacia la inmigración en educación física. Publicaciones, 49(4), 127-144. http:// doi.org/10.30827/publicaciones.v49i4.11732

León, O., Martínez, L. F. y Santos, M. L. (2018). Análisis de la investigación sobre Aprendizaje basado en Proyectos en Educación Física. Revista Electrónica Interuniversitaria de Formación del Profesorado, 21(2), 27-42.

Lorente, E. y Martos, D. (2018). Educación física y pedagogía crítica. Propuestas para la transformación personal y social. Lleida: Universidad de Valencia y Lleida.

Martí, M., Corbatón, R. y Serret, A. (2018). El Aprendizaje-Servicio: estrategias, procedimientos y acciones metodológicas. En O. Chiva-Bartoll y Gil-Gómez (Eds.), Aprendizaje-Servicio universitario: Modelos de intervención e investigación en la formación docente (pp. 25-36). Barcelona: Octaedro.

Mayor, D. (2019). Ejes pedagógicos que articulan los proyectos de Aprendizaje-Servicio. Revista Internacional de Educación y Aprendizaje, 7(1), 29-36. Recuperado de https://journals.epistemopolis.org/educacion/article/view/1940

Mayor, D. (2020). Aprendizaje-Servicio como estrategia metodológica para impulsar procesos de educación expandida. REMIE - Multidisciplinary Journal of Educational Research, 10(1), 48-75. http://doi.org/10.447/remie.2020.4562

Mayring, P. (2000). Qualitative content analysis. Forum qualitative social research, 1(2).

Meaney, K., Kopf, K., Bohler, H., Hernandez, L. y Scott, L. (2008). Service-learning and pre-service educators' cultural competence for teaching: An exploratory study. Journal of Experiential Education, 31, 189-208.

Melchior, A. (1999). Summary report: National evaluation of Learn and Serve America. Waltham: Center for Human Resources, Brandeis University.

Miles, M. y Huberman, A. M. (1984). Qualitative data analysis. A source book of new methods. Beverly Hills: Sage.

Páez, M. y Puig, J. M. (2013). La reflexión en el Aprendizaje-Servicio. Revista Internacional de Educación para la Justicia Social, 2(2), 13-32.

Pegalajar, M. C. (2018). Formación en competencias en alumnado universitario mediante prácticas basadas en aprendizaje cooperativo. Revista Complutense de Educación, 29(3), 35-52.

Puig, J. (Coord.). Aprendizaje-servicio (ApS). Educación y compromiso cívico. Barcelona: Graó.

Reyes, J. E. S. y Martínez, Y. B. (2019). El aprendizaje-servicio como aporte a la formación profesional: un análisis de caso a partir de la metodología Quinta Dimensión. Avances en Psicología Latinoamericana, 37(3), 541. 
Rodríguez, M. R. (2014). El Aprendizaje-Servicio como estrategia metodológica en la Universidad. Revista Complutense de Educación, 25, 95-113.

Romero, C., Zurita-Ortega, F. y Zurita-Molina, F. (2010). La autonomía y orientación en el Espacio Europeo de Educación Superior mediante el portafolio y la tutoría. Estudios sobre Educación, 19, 261-282.

Rubio, L. y Escofet, A. (Coords.) (2017). Aprendizaje-servicio (ApS): claves para su desarrollo en la universidad. Barcelona: Octaedro.

Ruiz-Corbella, M. y García-Gutiérrez, J. (2019). Aprendizaje-Servicio. Los retos de la evaluación. Madrid: Narcea.

Sanders, M. J., Vans, T. y McGeary, S. (2015). Analyzing Reflections in Service Learning to Promote Personal Growth and Community Self-Efficacy. Journal of Experiential Education, 39, 73-88.

Santos, M. A., Sotelino, A. y Lorenzo, M. (2015). Aprendizaje-servicio y misión cívica de la universidad. Una propuesta de desarrollo. Barcelona: Octaedro.

Santos, M. L., Martínez, L. F. y Cañadas, L. (2020). Limitaciones del aprendizajeservicio en la formación inicial en actividad físico-deportiva. Retos. Nuevas tendencias en Educación Física, Deporte y Recreación, 37, 567-575.

Smit, E. M. (2017). Value of Online Group Reflection After Internacional ServiceLearning Experiences. Nurse Educador, 42, 286-289.

Tejada-Fernández, J. y Ruiz-Bueno, C. (2016). Evaluación de competencias profesionales en educación superior: Retos e implicaciones. Educación XXI, 19(1), 17-37. http://doi.org/10.5944/ educxx1.12175

Wang, J., Lin, E., Spalding, E., Klecka, C. y Odell, S. (2011). Quality Teaching and Teacher Education: A Kaleidoscope of Notions. Journal of Teacher Education, 62(4), 331-338. 